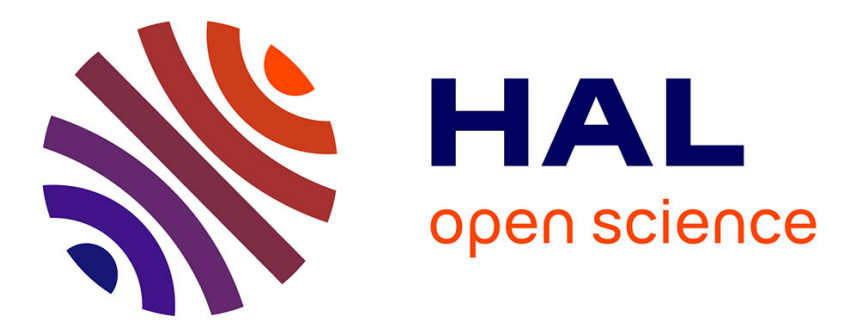

\title{
Constitutive model for flake graphite cast iron automotive brake discs: induced anisotropic damage model under complex loadings
}

\author{
L Augustins, René Billardon, François Hild
}

\section{- To cite this version:}

L Augustins, René Billardon, François Hild. Constitutive model for flake graphite cast iron automotive brake discs: induced anisotropic damage model under complex loadings. Continuum Mechanics and Thermodynamics, 2016, 28, pp.1445 - 1460. 10.1007/s00161-015-0487-5 . hal-01383893

\section{HAL Id: hal-01383893 \\ https://hal.science/hal-01383893}

Submitted on 19 Oct 2016

HAL is a multi-disciplinary open access archive for the deposit and dissemination of scientific research documents, whether they are published or not. The documents may come from teaching and research institutions in France or abroad, or from public or private research centers.
L'archive ouverte pluridisciplinaire HAL, est destinée au dépôt et à la diffusion de documents scientifiques de niveau recherche, publiés ou non, émanant des établissements d'enseignement et de recherche français ou étrangers, des laboratoires publics ou privés. 


\title{
Constitutive model for flake graphite cast iron automotive brake discs
}

\section{Induced anisotropic damage model under complex loadings}

\author{
L. Augustins · R. Billardon · F. Hild
}

Received: date / Accepted: date

\begin{abstract}
The present paper details an elasto-viscoplastic constitutive model for automotive brake discs made of flake graphite cast iron. In a companion paper [2], the authors proposed a one-dimensional setting appropriate for representing the complex behavior of the material (i.e. asymmetry between tensile and compressive loadings) under anisothermal conditions. The generalization of this $1 \mathrm{D}$ model to $3 \mathrm{D}$ cases on a volume element and the associated challenges are addressed. A direct transposition is not possible and an alternative solution without unilateral conditions is first proposed. Induced anisotropic damage and associated constitutive laws are then introduced. The transition from the volume element to the real structure and the numerical implementation requires a specific basis change. Brake disc simulations with this constitutive model show that unilateral conditions are needed for the friction bands. A damage deactivation procedure is therefore defined.
\end{abstract}

Keywords Flake graphite cast iron - Constitutive model · Brake discs . Anisotropic damage · Damage deactivation

\section{Introduction}

The present paper is the second part of a study devoted to the simulation of the thermomechanical behavior of automotive brake discs made of flake graphite

\author{
L. Augustins \\ PSA Peugeot Citroën \\ Route de Gisy, 78943 Velizy-Villacoublay Cedex, France \\ E-mail: louis.augustins@mpsa.com \\ R. Billardon \\ Messier-Bugatti-Dowty \\ Inovel Parc Sud, 78140 Vélizy-Villacoublay, France \\ E-mail: rene.billardon@safranmbd.com \\ F. Hild \\ LMT Cachan, ENS Cachan/CNRS/University Paris Saclay \\ 61 avenue du président Wilson, 94235 Cachan cedex, France \\ E-mail: hild@lmt.ens-cachan.fr
}


(grey) cast iron (i.e. FGCI). An overview of the industrial and scientific issues mainly related to the thermomechanical fatigue design of automotive brake discs and to the correct description of the properties of their constitutive material - is proposed in the first part [2].

The model developed earlier is based on an elasto-viscoplastic postulate with the formalism proposed by Chaboche [7], and on a precise description of the graphite flakes opening and closure phases, which are responsible for the strong dissymmetry between tensile and compressive response of FGCI [21]. Such a model allows for an accurate simulation of the cyclic behavior of FGCI in isothermal or anisothermal conditions for a one-dimensional tension/compression loading.

Automotive brake discs are subjected to severe thermomechanical loadings [15, $1,39]$ leading to cyclic plasticity in the grooves and potential crack initiation [39, 16]. Three-dimensional finite element calculations are necessary to assess the physical quantities that control the fatigue life of such a structure. As a one-dimensional description of the material was proposed [2], a 3D extension applicable under complex loadings needs to be formulated.

In the present paper, the generalization of the $1 \mathrm{D}$ model to $3 \mathrm{D}$ cases on a volume element is addressed as a first step. The transition from this volume element to the real structure and the corresponding numerical implementation are then described.

\section{From a 1D description to a $3 \mathrm{D}$ constitutive model}

\subsection{Extension of the rheological model}

A complete 3D braking simulation requires the $1 \mathrm{D}$ model proposed by the authors [2] to be extended to 3D situations, which are valid under complex loading histories. This generalization is not straight forward:

- Superposition of 1D models composed of two $1 D$ branches (i.e. one for the damaged matrix and one for the debonded flakes) in the three principal directions. When the stress tensor has more than one component, reducing the three-dimensional formulation to a one-dimensional expression proves impossible. Since there is no reason for the stress tensor to have only one component in a $3 \mathrm{D}$ structure subjected to complex loads, this option must be disregarded.

- Extension of the 1D model [2] to a constitutive law containing two 3D branches. The objective of the present work is to implement the constitutive model in the finite element solver ABAQUS using a user material subroutine (UMAT [37]). The input data to this type of routine are the total strain tensor increment, and the internal variables at the beginning of the increment. If, for instance, a tensile loading in direction 1 is considered, the global strain increments will have three non zero terms, namely, $\Delta \epsilon_{11}$, and $\Delta \epsilon_{22}=\Delta \epsilon_{33}=-\nu \Delta \epsilon_{11}$. The Poisson's ratio $\nu$ is differently affected by damage in the two branches $(D$ and $(1-D))$. As a consequence, there is an incompatibility between the transverse strains $\left(\epsilon_{22}\right.$ and $\left.\epsilon_{33}\right)$ of the two branches. This option entails an over or underestimation of the stresses in one of the branches.

It is therefore worth asking whether managing the opening/closure mechanisms is crucial to a specific application on brake discs, and to what extent accurately 
simulating the behavior of the disc without this phenomenon is possible. To answer this question, it is necessary to study the stress/strain paths in the critical areas of the disc, and to understand the conditions under which the flakes open and close within the structure. A 3D constitutive model that can take into account flake debonding induced by loading and the associated anisotropic damage description is required. This model will represent flake opening but not its closure, a pattern that corresponds to the matrix branch in the 1D model [2], and will make it possible to say whether debonded flakes fulfill the conditions under which they will close on a real structure.

\section{$2.23 \mathrm{D}$ Constitutive model without flake closure}

\subsubsection{Anisotropic damage}

Any damage orientation distribution can be theoretically considered, resulting in an anisotropic damage description [25,31]. However, a preferred orientation of microcracking, more or less perpendicular to the maximum principal stress, is more often the case [28]. The simplest way to represent damage without referring to a particular basis (i.e. intrinsic writing) is to resort to even order tensors [25].

The most general form that allows the basis change invariance for any damage distribution to be satisfied requires the use of an eighth order tensor [7]. Many authors prefer simpler models and favor fourth order $[8,34,24]$ or second order [33, $23,10,9,22,14$ ] tensors, by which complex anisotropy problems and simple damage symmetries such as orthotropy are described.

In this study, damage is induced by the loading and corresponds to graphite flakes debonding, which is mostly oriented perpendicular to the loading direction. Figure 1 illustrates this effect by showing an idealized distribution composed of planar flakes. Given that the loading on a brake disc is known, the selected option is to represent damage by a second order tensor via the introduction of the degradation of the elastic behavior [10].
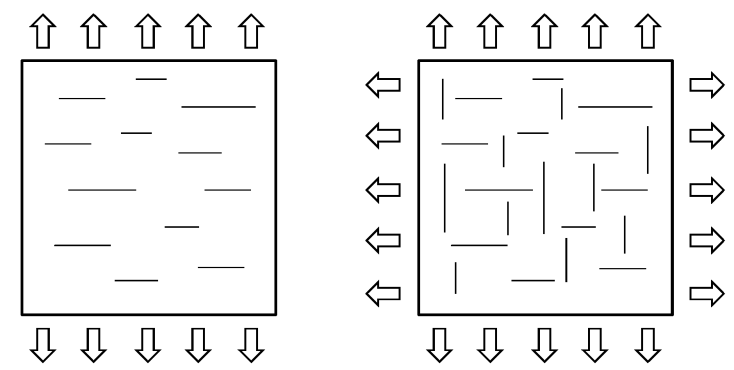

Fig. 1: Schematic drawing of induced damage

The form of the damaged material compliance tensor $\widetilde{\mathbb{S}}$ is derived from Refs. [13, 18], itself derived from Fabrikant's work [17]. The authors studied unidirectional 
crack distributions perpendicular to the eigen directions in an isotropic elastic matrix. If only the distribution perpendicular to the 1-axis is activated, the following results are obtained

$$
\begin{gathered}
\frac{E_{1}}{E_{0}}=(1-D) \quad, \quad \frac{E_{2}}{E_{0}}=\frac{E_{3}}{E_{0}}=\frac{\nu_{23}}{\nu_{0}}=1 \\
\frac{\nu_{12}}{\nu_{0}}=\frac{\nu_{13}}{\nu_{0}}=\frac{E_{1}}{E_{0}} \quad, \quad \frac{G_{12}}{G_{0}}=\frac{G_{13}}{G_{0}}=(1-D)^{\alpha}
\end{gathered}
$$

where subscripts 0 correspond to the initial isotropic matrix, $E, G$ and $\nu$ to Young's modulus, shear modulus and Poisson's ratio, and $D$ to the damage variable. In Equation (2), a power $\alpha$ is introduced and is equal to 0.5 [36]. The superposition principle then makes it possible to define a compliance tensor for the damaged material involving three scalar damage variables associated with three crack distributions

$\widetilde{\mathbb{S}}=\widetilde{\mathbb{C}}^{-1}=\frac{1}{E}\left(\begin{array}{cccccc}\frac{1}{1-D_{1}} & -\nu & -\nu & 0 & 0 & 0 \\ & \frac{1}{1-D_{2}} & -\nu & 0 & 0 & 0 \\ & & \frac{1}{1-D_{3}} & 0 & 0 & 0 \\ & \text { sym. } & & \frac{1+\nu}{\sqrt{\left(1-D_{1}\right)\left(1-D_{2}\right)}} & 0 & 0 \\ & & & & \frac{1+\nu}{\sqrt{\left(1-D_{2}\right)\left(1-D_{3}\right)}} & 0 \\ & & & & & \frac{1+\nu}{\sqrt{\left(1-D_{1}\right)\left(1-D_{3}\right)}}\end{array}\right)$

where $\widetilde{\mathbb{C}}$ is Hooke's tensor of the damaged material. In the sequel, the notation $\mathbb{C}$ refers to a fourth-order tensor. This relationship is written in the eigen stress basis, which corresponds to the damage basis. As shown in the sequel, this setting proves sufficient for brake discs because damage anisotropy is induced by loading whose eigen directions do not change with time. This result is true in the specific problem discussed in the present work because of several features. In the most general case of a disc subjected to acceleration/breaking cycles, body forces induce shear stresses. The friction between the pad and the disc also results in a shear stress on the friction band. Models were proposed to solve such cases with an orthotropic damage growth rule applied to variable principal directions [38]. However, in the case of automotive grey cast iron brake discs, the stress levels generated by the heat flux on the friction band are significantly higher than the aforementioned stresses that are neglected herein.

\subsubsection{Effective stress, yield criterion}

The effective stress tensor $\tilde{\boldsymbol{\sigma}}$ is defined by the strain equivalence principle $[6,27]$ and corresponds to the stress that must be applied on an undamaged elastic volume element to obtain the same strain as that of a damaged element. This definition makes explicit the effective stress versus stress tensor $\boldsymbol{\sigma}$

$$
\tilde{\boldsymbol{\sigma}}=\mathbb{C}: \widetilde{\mathbb{S}}: \boldsymbol{\sigma}
$$

where $\mathbb{C}$ is Hooke's tensor for the undamaged material. 
The equivalent Von Mises stress, for the undamaged material, is then defined by

$$
\sigma_{e q}=\sqrt{\frac{3}{2} s: s}
$$

where $s$ is the deviatoric stress tensor

$$
s=\mathbb{K}: \sigma
$$

where $\mathbb{K}$ is the projector operator onto the deviatoric space

$$
\mathbb{K}=\mathbb{I}-\frac{1}{3} \mathbf{1} \otimes \mathbf{1}
$$

1 and $\mathbb{I}$ the second and fourth order identity tensors, respectively.

The equivalent stress of the damaged material is defined on the assumption that the first yielding state of the damaged material under a stress $\sigma$ is equivalent to that of the undamaged material under a stress $\tilde{\boldsymbol{\sigma}}$

$$
\tilde{\sigma}_{e q}=\sqrt{\frac{3}{2} \tilde{\boldsymbol{s}}: \tilde{\boldsymbol{s}}}=\sqrt{\frac{3}{2}(\mathbb{K}: \tilde{\boldsymbol{\sigma}}):(\mathbb{K}: \tilde{\boldsymbol{\sigma}})}
$$

with $\mathbb{M}=\mathbb{K}: \mathbb{C}: \widetilde{\mathbb{S}}$ and given Equation (4), it leads to

$$
\tilde{\sigma}_{e q}=\sqrt{\frac{3}{2}(\mathbb{M}: \sigma):(\mathbb{M}: \sigma)}
$$

\subsubsection{Thermodynamic framework}

In this section, the thermodynamic framework for the undamaged material is defined within the generalized standard material setting [20] with a unified viscoplasticity model [7]. The state potential is defined by the free energy density

$$
\rho \omega=\frac{1}{2}\left(\boldsymbol{\epsilon}-\boldsymbol{\epsilon}_{t h}-\boldsymbol{\epsilon}_{v p}\right): \mathbb{C}:\left(\boldsymbol{\epsilon}-\boldsymbol{\epsilon}_{t h}-\boldsymbol{\epsilon}_{v p}\right)+\omega_{p}(\boldsymbol{\alpha})
$$

where $\omega_{p}(\boldsymbol{\alpha})=\frac{1}{3} C \boldsymbol{\alpha}: \boldsymbol{\alpha}$ is the energy stored in hardenings. The dual dissipation potential is chosen as

$$
\phi^{\star}=\frac{K}{m+1}\left\langle\frac{f}{K}\right\rangle^{m+1}
$$

where $K$ and $m$ are Norton's viscous parameters, and $f$ the yield surface.

In order to simplify these equations, only one nonlinear kinematic hardening term is considered in the present section. This hypothesis implies a flow potential different from $f$ (i.e. non-associated plasticity [28])

$$
F=f+\frac{3 \gamma}{4 C} \boldsymbol{X}: \boldsymbol{X}
$$

with

$$
f=(\boldsymbol{\sigma}-\boldsymbol{X})_{e q}-\sigma_{0}
$$


where $C$ is the hardening modulus, and $\gamma$ the inverse of a characteristic strain associated with the nonlinearity of the hardening. Applying the normality rule, the growth laws read

$$
\begin{gathered}
\dot{\boldsymbol{\epsilon}}_{v p}=\frac{\partial \phi^{\star}}{\partial \boldsymbol{\sigma}}=\dot{\lambda} \frac{\partial F}{\partial \boldsymbol{\sigma}}=\left\langle\frac{f}{K}\right\rangle^{m} \frac{3}{2} \frac{(\boldsymbol{s}-\boldsymbol{X})}{(\boldsymbol{\sigma}-\boldsymbol{X})_{e q}} \\
\dot{\boldsymbol{\alpha}}=\frac{\partial \phi^{\star}}{\partial \boldsymbol{X}}=\dot{\lambda} \frac{\partial F}{\partial \boldsymbol{X}}=-\dot{\boldsymbol{\epsilon}}_{v p}-\dot{p} \gamma \boldsymbol{\alpha}
\end{gathered}
$$

The cumulated plastic strain rate $\dot{p}$ is deduced from energy equivalence

$$
(s-\boldsymbol{X}): \dot{\boldsymbol{\epsilon}}_{v p}=(\boldsymbol{\sigma}-\boldsymbol{X})_{e q} \dot{p}
$$

and corresponds to the viscoplastic multiplier

$$
\dot{p}=\dot{\lambda}=\left\langle\frac{f}{K}\right\rangle^{m}=\sqrt{\frac{2}{3} \dot{\boldsymbol{\epsilon}}_{v p}: \dot{\boldsymbol{\epsilon}}_{v p}}
$$

\subsubsection{State and growth laws}

In this subsection, state and growth laws are introduced for the damaged material on the assumption of a state decoupling between plasticity and damage [32]. The thermodynamic potential is written in an analogous way as Equation (10), with the difference that $\widetilde{\mathbb{C}}$ is substituted for $\mathbb{C}$

$$
\rho \omega=\frac{1}{2}\left(\boldsymbol{\epsilon}-\boldsymbol{\epsilon}_{t h}-\boldsymbol{\epsilon}_{v p}\right): \widetilde{\mathbb{C}}:\left(\boldsymbol{\epsilon}-\boldsymbol{\epsilon}_{t h}-\boldsymbol{\epsilon}_{v p}\right)+\omega_{p}\left(\boldsymbol{\alpha}_{i}\right)
$$

where the viscoplasticity related terms remain unchanged. The state laws then read

$$
\begin{gathered}
\boldsymbol{\sigma}=\rho \frac{\partial \omega}{\partial \boldsymbol{\epsilon}} \\
\boldsymbol{X}_{i}=-\rho \frac{\partial \omega}{\partial \boldsymbol{\alpha}_{i}}
\end{gathered}
$$

The first back-stress $\boldsymbol{X}_{1}$ is nonlinear and the second one $\boldsymbol{X}_{2}$ is linear. The thermodynamic force $Y$ associated with $D$ is defined as

$$
Y=-\rho \frac{\partial \omega}{\partial D}
$$

It should be emphasized that the damage variable is introduced in the model to simulate the stabilized behavior of the material and not to calculate continuous damage right up to material failure. The growth of $D$ must therefore be bounded by a maximum value - potentially reached after a first tensile phase - associated with the volume fraction of debonded graphite flakes. Under these conditions, in order to have more freedom in the definition of the damage growth law, the latter is written in a non standard form detailed in Ref. [2]. The flow potential $F$ now reads

$$
F=\left(\tilde{\boldsymbol{\sigma}}-\sum_{i} \boldsymbol{X}_{i}\right)_{e q}-\sigma_{0}+\frac{3 \gamma}{4 C_{1}}\left(\boldsymbol{X}_{1}: \boldsymbol{X}_{1}-\frac{4}{9} C_{1}^{2} \boldsymbol{\alpha}_{1}: \boldsymbol{\alpha}_{1}\right)
$$


with

$$
\left(\tilde{\boldsymbol{\sigma}}-\sum_{i} \boldsymbol{X}_{i}\right)_{e q}=\sqrt{\frac{3}{2}\left(\mathbb{M}: \boldsymbol{\sigma}-\sum_{i} \boldsymbol{X}_{i}\right):\left(\mathbb{M}: \boldsymbol{\sigma}-\sum_{i} \boldsymbol{X}_{i}\right)}=\mathcal{J}
$$

and $\boldsymbol{X}_{i}=-\frac{2}{3} C \boldsymbol{\alpha}_{i}$.

The growth laws for the damaged material become

$$
\dot{\boldsymbol{\epsilon}}_{v p}=\frac{\partial \phi^{\star}}{\partial \boldsymbol{\sigma}}=\dot{\lambda} \frac{\partial F}{\partial \boldsymbol{\sigma}}=\left\langle\frac{f}{K}\right\rangle^{m} \frac{3}{2} \frac{\left(\mathbb{M}^{T}: \mathbb{M}: \boldsymbol{\sigma}-\mathbb{M}^{T}: \sum_{i} \boldsymbol{X}_{i}\right)}{\left(\tilde{\boldsymbol{\sigma}}-\sum_{i} \boldsymbol{X}_{i}\right)_{e q}}=\left\langle\frac{f}{K}\right\rangle^{m} \boldsymbol{n}
$$

with

$$
\begin{gathered}
\boldsymbol{n}=\frac{3}{2} \frac{\left(\mathbb{M}^{T}: \mathbb{M}: \boldsymbol{\sigma}-\mathbb{M}^{T}: \sum_{i} \boldsymbol{X}_{i}\right)}{\left(\tilde{\boldsymbol{\sigma}}-\sum_{i} \boldsymbol{X}_{i}\right)_{e q}} \\
\dot{\boldsymbol{\alpha}}_{1}=\frac{\partial \phi^{\star}}{\partial \boldsymbol{X}_{1}}=\dot{\lambda} \frac{\partial F}{\partial \boldsymbol{X}_{1}}=-\left\langle\frac{f}{K}\right\rangle^{m}\left(\frac{3}{2} \frac{\left(\mathbb{M}: \boldsymbol{\sigma}-\sum_{i} \boldsymbol{X}_{i}\right)}{\left(\tilde{\boldsymbol{\sigma}}-\sum_{i} \boldsymbol{X}_{i}\right)_{e q}}+\gamma \boldsymbol{\alpha}_{1}\right)=\left\langle\frac{f}{K}\right\rangle^{m}\left(\boldsymbol{n}_{X}-\gamma \boldsymbol{\alpha}_{1}\right)
\end{gathered}
$$

and

$$
\begin{gathered}
\boldsymbol{n}_{X}=-\frac{3}{2} \frac{\left(\mathbb{M}: \boldsymbol{\sigma}-\sum_{i} \boldsymbol{X}_{i}\right)}{\left(\tilde{\boldsymbol{\sigma}}-\sum_{i} \boldsymbol{X}_{i}\right)_{e q}} \\
\dot{\boldsymbol{\alpha}}_{2}=\frac{\partial \phi^{\star}}{\partial \boldsymbol{X}_{2}}=\dot{\lambda} \frac{\partial F}{\partial \boldsymbol{X}_{2}}=-\left\langle\frac{f}{K}\right\rangle^{m}\left(\frac{3}{2} \frac{\left(\mathbb{M}: \boldsymbol{\sigma}-\sum_{i} \boldsymbol{X}_{i}\right)}{\left(\tilde{\boldsymbol{\sigma}}-\sum_{i} \boldsymbol{X}_{i}\right)_{e q}}\right)=\left\langle\frac{f}{K}\right\rangle^{m} \boldsymbol{n}_{X}
\end{gathered}
$$

"Auxiliary" variables are introduced via the use of "effective" viscoplastic strain and cumulated viscoplastic strain rate that help, in the anisotropic case, to significantly simplify the numerical implementation of the model. The "effective" viscoplastic strain is defined as

$$
\dot{\tilde{\boldsymbol{\epsilon}}}_{v p}=\frac{\partial \phi^{\star}}{\partial \tilde{\boldsymbol{\sigma}}}=\dot{\lambda} \frac{\partial F}{\partial \tilde{\boldsymbol{\sigma}}}=\left\langle\frac{f}{K}\right\rangle^{m} \frac{3}{2} \frac{\left(\tilde{\boldsymbol{s}}-\sum_{i} \boldsymbol{X}_{i}\right)}{\left(\tilde{\boldsymbol{\sigma}}-\sum_{i} \boldsymbol{X}_{i}\right)_{e q}}
$$

The "effective" cumulated viscoplastic strain rate $\dot{\tilde{p}}$ is deduced from energy equivalence

$$
\left(\tilde{\boldsymbol{s}}-\sum_{i} \boldsymbol{X}_{i}\right): \dot{\tilde{\boldsymbol{\epsilon}}}_{v p}=\left(\tilde{\boldsymbol{\sigma}}-\sum_{i} \boldsymbol{X}_{i}\right)_{e q} \dot{\tilde{p}}
$$


such that

$$
\dot{\tilde{p}}=\dot{\lambda}=\left\langle\frac{f}{K}\right\rangle^{m}=\sqrt{\frac{2}{3} \dot{\tilde{\boldsymbol{\epsilon}}}_{v p}: \dot{\tilde{\boldsymbol{\epsilon}}}_{v p}}
$$

Equation (29) shows that the viscous stress is a function of the "effective" cumulated viscoplastic strain rate rather than the cumulated viscoplastic strain rate.

\subsubsection{Numerical integration}

The constitutive model was implemented using an implicit integration scheme [35] in an ABAqus UMAT routine [37]. It is a generalized middle point $\theta$-method [29] with $\theta=1$ (i.e. fully implicit Euler method). The solution to the numerical problem in structural mechanics consists of calculating the stress increment $\Delta \sigma$ induced by a total strain increment $\Delta \epsilon$ prescribed during $\Delta t$.

Newton's method numerically minimizes the local residual defined as

$$
\{R\}=\left\{\Delta v_{i}\right\}-\Delta t\left\{\dot{v}_{i}(t+\theta \Delta t)\right\}
$$

The chosen integration variables are $\left\{\boldsymbol{\epsilon}_{e}, \boldsymbol{\alpha}_{1}, \boldsymbol{\alpha}_{2}, \tilde{p}\right\}$ so that the residual vector components are expressed as

$$
\begin{gathered}
\boldsymbol{R}_{\epsilon_{e}}=\Delta \boldsymbol{\epsilon}_{e}-(\Delta \boldsymbol{\epsilon}-\Delta \tilde{p} \boldsymbol{n}) \\
R_{\tilde{p}}=\Delta \tilde{p}-\Delta t\left\langle\frac{\mathcal{J}-\sigma_{0}}{K}\right\rangle^{m} \\
\boldsymbol{R}_{\alpha_{1}}=\Delta \boldsymbol{\alpha}_{1}-\Delta \tilde{p}\left(\boldsymbol{n}_{X}-\gamma \boldsymbol{\alpha}_{1}\right) \\
\boldsymbol{R}_{\alpha_{2}}=\Delta \boldsymbol{\alpha}_{2}-\Delta \tilde{p} \boldsymbol{n}_{X}
\end{gathered}
$$

The nonlinear problem to be solved is a $19 \times 19$ dimension system. The solution using Newton's method requires knowing the Jacobian matrix $\boldsymbol{J}$ of the system, which is determined analytically and defined by components such that

$$
J_{i j}=\frac{\partial R_{i}}{\partial \Delta v_{j}}
$$

It is written as

$$
\boldsymbol{J}=\left(\begin{array}{llll}
\frac{\partial \boldsymbol{R}_{\epsilon_{e}}}{\partial \Delta \boldsymbol{\epsilon}_{e}} & \frac{\partial \boldsymbol{R}_{\epsilon_{e}}}{\partial \Delta \boldsymbol{\alpha}_{1}} & \frac{\partial \boldsymbol{R}_{\epsilon_{e}}}{\partial \Delta \boldsymbol{\alpha}_{2}} & \frac{\partial \boldsymbol{R}_{\epsilon_{e}}}{\partial \Delta \tilde{p}} \\
\frac{\partial \boldsymbol{R}_{\alpha_{1}}}{\partial \Delta \boldsymbol{\epsilon}_{e}} & \frac{\partial \boldsymbol{R}_{\alpha_{1}}}{\partial \Delta \boldsymbol{\alpha}_{1}} & \frac{\partial \boldsymbol{R}_{\alpha_{1}}}{\partial \Delta \boldsymbol{\alpha}_{2}} & \frac{\partial \boldsymbol{R}_{\alpha_{1}}}{\partial \Delta \tilde{p}} \\
\frac{\partial \boldsymbol{R}_{\alpha_{2}}}{\partial \Delta \boldsymbol{\epsilon}_{e}} & \frac{\partial \boldsymbol{R}_{\alpha_{2}}}{\partial \Delta \boldsymbol{\alpha}_{1}} & \frac{\partial \boldsymbol{R}_{\alpha_{2}}}{\partial \Delta \boldsymbol{\alpha}_{2}} & \frac{\partial \boldsymbol{R}_{\alpha_{2}}}{\partial \Delta \tilde{p}} \\
\frac{\partial R_{\tilde{p}}}{\partial \Delta \boldsymbol{\epsilon}_{e}} & \frac{\partial R_{\tilde{p}}}{\partial \Delta \boldsymbol{\alpha}_{1}} & \frac{\partial R_{\tilde{p}}}{\partial \Delta \boldsymbol{\alpha}_{2}} & \frac{\partial R_{\tilde{p}}}{\partial \Delta \tilde{p}}
\end{array}\right)
$$

In order to express each term of the matrix, several quantities are defined

$$
\begin{gathered}
\mathbb{N}=\frac{\partial \boldsymbol{n}}{\partial \boldsymbol{\sigma}}=\frac{1}{\mathcal{J}}\left(\frac{3}{2} \mathbb{M}^{T}: \mathbb{M}-\boldsymbol{n} \otimes \boldsymbol{n}\right) \\
\mathbb{S}=\frac{\partial \boldsymbol{n}}{\partial \boldsymbol{X}}=-\frac{1}{\mathcal{J}}\left(\frac{3}{2} \mathbb{M}^{T}+\boldsymbol{n} \otimes \boldsymbol{n}_{X}\right) \\
\mathbb{N}_{X}=\frac{\partial \boldsymbol{n}_{X}}{\partial \boldsymbol{\sigma}}=-\frac{1}{\mathcal{J}}\left(\frac{3}{2} \mathbb{M}-\boldsymbol{n}_{X} \otimes \boldsymbol{n}\right)
\end{gathered}
$$




$$
\mathbb{S}_{X}=\frac{\partial \boldsymbol{n}_{X}}{\partial \boldsymbol{X}}=\frac{1}{\mathcal{J}}\left(\frac{3}{2} \mathbb{K}-\boldsymbol{n}_{X} \otimes \boldsymbol{n}_{X}\right)
$$

The terms derived from Equation (31) read

$$
\begin{gathered}
\frac{\partial \boldsymbol{R}_{\epsilon_{e}}}{\partial \Delta \boldsymbol{\epsilon}_{e}}=\mathbb{I}+\theta \Delta \tilde{p} \mathbb{N}: \widetilde{\mathbb{C}} \\
\frac{\partial \boldsymbol{R}_{\epsilon_{e}}}{\partial \Delta \tilde{p}}=\boldsymbol{n} \\
\frac{\partial \boldsymbol{R}_{\epsilon_{e}}}{\partial \Delta \boldsymbol{\alpha}_{1}}=-\frac{2}{3} C_{1} \theta \Delta \tilde{p} \mathbb{S} \\
\frac{\partial \boldsymbol{R}_{\epsilon_{e}}}{\partial \Delta \boldsymbol{\alpha}_{2}}=-\frac{2}{3} C_{2} \theta \Delta \tilde{p} \mathbb{S}
\end{gathered}
$$

When the viscoplastic flow is activated $\left(\mathcal{J}>\sigma_{0}\right)$, the terms derived from Equation (32) become

$$
\begin{gathered}
\frac{\partial R_{\tilde{p}}}{\partial \Delta \boldsymbol{\epsilon}_{e}}=-\frac{m}{K} \theta \Delta \tilde{p}\left\langle\frac{\mathcal{J}-\sigma_{0}}{K}\right\rangle^{m-1} \boldsymbol{n}: \widetilde{\mathbb{C}} \\
\frac{\partial R_{\tilde{p}}}{\partial \Delta \tilde{p}}=1 \\
\frac{\partial R_{\tilde{p}}}{\partial \Delta \boldsymbol{\alpha}_{1}}=\frac{m}{K} \theta \Delta \tilde{p}\left\langle\frac{\mathcal{J}-\sigma_{0}}{K}\right\rangle^{m-1} \frac{2 C_{1}}{3} \boldsymbol{n}_{X} \\
\frac{\partial R_{\tilde{p}}}{\partial \Delta \boldsymbol{\alpha}_{2}}=\frac{m}{K} \theta \Delta \tilde{p}\left\langle\frac{\mathcal{J}-\sigma_{0}}{K}\right\rangle^{m-1} \frac{2 C_{2}}{3} \boldsymbol{n}_{X}
\end{gathered}
$$

and for hardening variables (see Equations. (33) and (34))

$$
\begin{gathered}
\frac{\partial \boldsymbol{R}_{\alpha_{1}}}{\partial \Delta \boldsymbol{\epsilon}_{e}}=-\theta \Delta \tilde{p} \mathbb{N}_{X}: \widetilde{\mathbb{C}} \\
\frac{\partial \boldsymbol{R}_{\alpha_{1}}}{\partial \Delta \tilde{p}}=-\boldsymbol{n}_{X}+\gamma \boldsymbol{\alpha}_{1} \\
\frac{\partial \boldsymbol{R}_{\alpha_{1}}}{\partial \Delta \boldsymbol{\alpha}_{1}}=\mathbb{I}+\theta \Delta \tilde{p}\left(\frac{2 C_{1}}{3} \mathbb{S}_{X}+\gamma \mathbb{I}\right) \\
\frac{\partial \boldsymbol{R}_{\alpha_{1}}}{\partial \Delta \boldsymbol{\alpha}_{2}}=\frac{2}{3} C_{2} \theta \Delta \tilde{p} \mathbb{S}_{X}
\end{gathered}
$$

and

$$
\begin{gathered}
\frac{\partial \boldsymbol{R}_{\alpha_{2}}}{\partial \Delta \boldsymbol{\epsilon}_{e}}=-\theta \Delta \tilde{p} \mathbb{N}_{X}: \widetilde{\mathbb{C}} \\
\frac{\partial \boldsymbol{R}_{\alpha_{2}}}{\partial \Delta \tilde{p}}=-\boldsymbol{n}_{X} \\
\frac{\partial \boldsymbol{R}_{\alpha_{2}}}{\partial \Delta \boldsymbol{\alpha}_{1}}=\frac{2}{3} C_{1} \theta \Delta \tilde{p} \mathbb{S}_{X} \\
\frac{\partial \boldsymbol{R}_{\alpha_{2}}}{\partial \Delta \boldsymbol{\alpha}_{2}}=\mathbb{I}+\frac{2}{3} C_{2} \theta \Delta \tilde{p} \mathbb{S}_{X}
\end{gathered}
$$


The stress increment $\Delta \boldsymbol{\sigma}$ is calculated from the elastic strain increment $\Delta \boldsymbol{\epsilon}_{e}$ when Newton's method has converged. The variation of the damaged Hooke's tensor $\widetilde{\mathbb{C}}$ must also be considered. The implicit integration scheme leads to

$$
\Delta \boldsymbol{\sigma}=\dot{\boldsymbol{\sigma}}(t+\theta \Delta t) \Delta t
$$

which is developed as

$$
\Delta \boldsymbol{\sigma}=\widetilde{\mathbb{C}}: \Delta \boldsymbol{\epsilon}_{e}+\Delta \widetilde{\mathbb{C}}: \boldsymbol{\epsilon}_{e}
$$

It is useful to define the consistent tangent matrix linked to the integration scheme in order to optimize the global convergence and avoid too small time increments. This operator is defined by

$$
\boldsymbol{L}=\frac{\partial \Delta \boldsymbol{\sigma}}{\partial \Delta \boldsymbol{\epsilon}}
$$

It can be shown that [5]

$$
\boldsymbol{L}=\widetilde{\mathbb{C}}: \boldsymbol{J}_{e e}^{*}
$$

where $\boldsymbol{J}_{e e}^{*}$ is defined from the inverse of the Jacobian $\boldsymbol{J}^{-1}$. It is the block that corresponds to the subscripts linked to the elastic strain $\boldsymbol{\epsilon}_{e}$ tensor.

Although the application of non-associated plasticity $(f \neq F)$ results in a lack of symmetry of the tangent operator, no particular problems of convergence were observed on the complex 3D FE problems considered herein. Furthermore, convergence with the calculated elastoplastic tangent matrix is far better than that observed with a simple elastic one.

\section{From a volume element to a structure}

The constitutive model presented in Section 2.2, which is valid for monotonic loading histories (i.e. without flake closure) on a volume element, must now be extended to complex loadings in a structure. The main challenge is to deal with the damage directions, namely, the description of the damaged compliance tensor $\widetilde{\mathbb{S}}$ defined in Equation (3) implies that the constitutive equations be written in the damage basis, which corresponds to the eigen basis assumed to remain unchanged with time.

In this section, the architecture of a brake disc system and the damage mechanisms on critical areas are briefly described. Then, following a presentation of the heat flux modeling, the implementation of the model is described taking into account the previous challenges. Brake disc simulations, on which the flake closure conditions are studied, are then carried out. Model parameters are those identified in Ref. [2]. A solution is finally proposed to deal with flake opening and closure in specific areas of the disc.

\subsection{Architecture of a brake disc system}

Brake disc systems appeared in the 1950 s to overcome the drawbacks of drum brakes. Such systems are now widely used in the automotive as well as in the rail and aeronautical industries. The system, which is shown in Figure 2, includes the following elements: 
- A plain or ventilated disc, generally made of cast iron, connected to the rim by means of the hub. As a rotating part, its role is to absorb and dissipate the mechanical energy transformed into heat during braking.

- Two brake pads, made of frictional material (the friction lining) mounted on a metallic support. The pads are brought into contact with the disc during braking.

- A caliper, a motionless part generally connected to the steering knuckle, on which the pads are attached. Yoke shaped, it covers an angular sector of the disc. Its role is to press the pads against the disc to slow it down. The caliper is equipped with one or more hydraulic pistons.

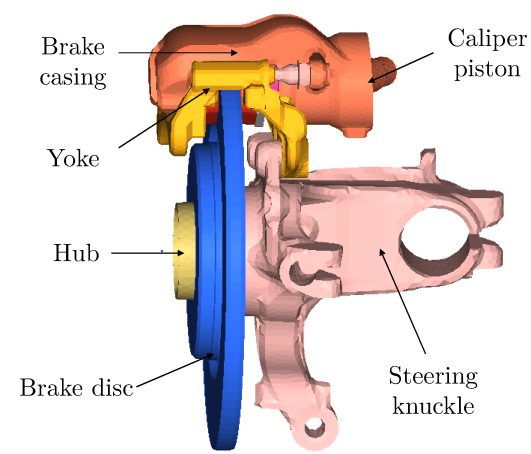

(a) Full view

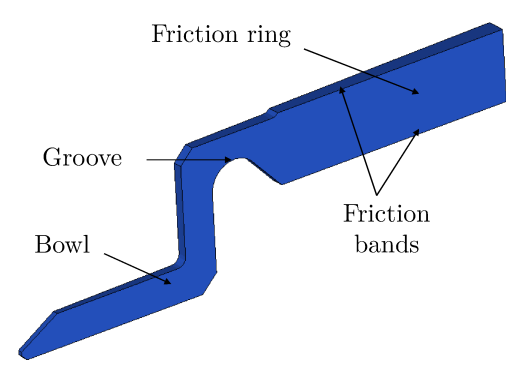

(b) Plain brake disc

Fig. 2: Brake disc system

\subsection{Damage mechanisms}

Damage inducing fatigue cracks on a brake disc is mainly due to the presence of high thermal gradients that occur during braking. As a consequence, the disc tends to expand in the hot zones, but neighboring cold zones prevent free expansion. There are two main types of thermal gradients:

- the gradient between the friction ring, which is directly affected by the heat flux, and the bowl;

- the gradient between the friction bands.

The gradient between the friction ring and the bowl (see Figure 3) gives rise to "coning" [26], namely, an axial distortion of the brake disc planar geometry, which results in the disc becoming conical in shape, owing to the different thermal expansions. The groove is thus subjected to high tensile stresses during braking leading to local plastic strains. Upon cooling, the friction ring straightens without returning to its original position in conjunction with the occurrence of compressive 
plasticity in the same zone. The area is thus subjected to cyclic plasticity and can lead, on severe bench tests, to circumferential cracks revealed in Figure 4(a) with dye penetrant.

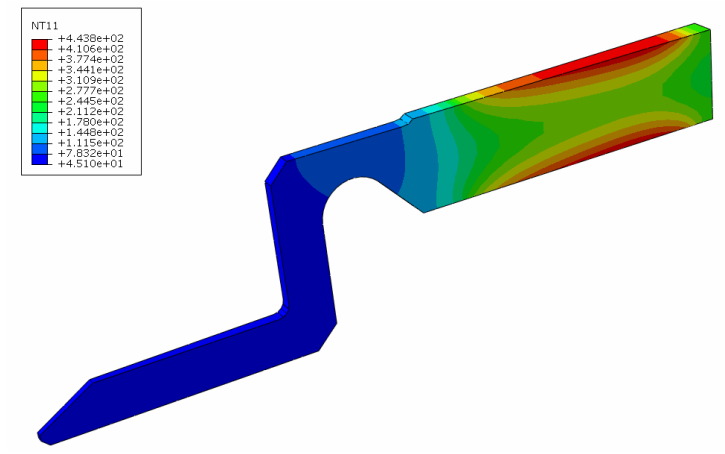

Fig. 3: Temperature field at maximum temperature time of the friction bands

The gradient between the friction bands (see Figure 3) is explained by the following sequence. As breaking generally lasts only a few seconds, the friction bands are heated very quickly. The surface temperature can reach up to $600{ }^{\circ} \mathrm{C}$ in 4 or $5 \mathrm{~s}$, while the center of the friction ring only reaches about 200 or $300{ }^{\circ} \mathrm{C}$ during the same time. This difference in temperature with depth induces high levels of radial and hoop compressive stresses. Upon cooling, residual tensile stresses following plastic compressive strains appear. On severe bench tests, this phenomenon leads to thermal striping $[12,4]$ in the form of radial cracks on friction bands (Figure $4(\mathrm{~b}))$.

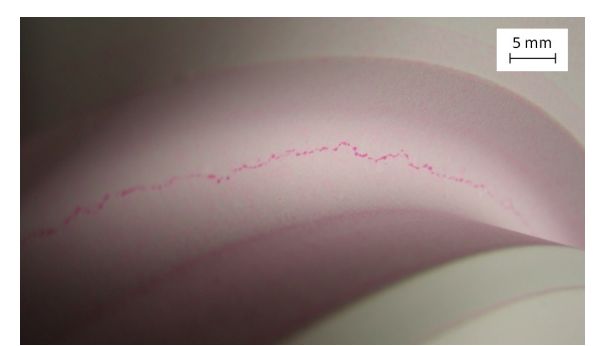

(a) Circumferential crack in a groove

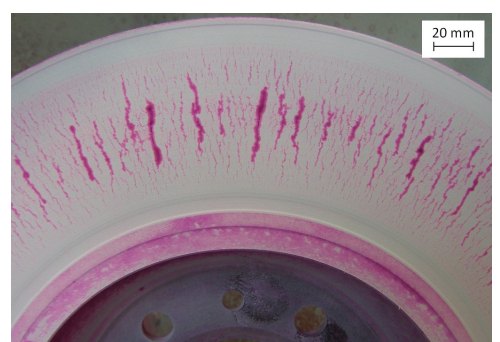

(b) Radial cracks in a friction band

Fig. 4: Cracked areas on a brake disc subjected to severe bench test

\subsection{External load description}

As a first approximation, the intensity of the heat flux experienced by the disc directly depends on the lining pressure field $P$, the friction coefficient $\mu$ between 
the disc and the linings, the wheel angular velocity $\omega$ and $r$ the radius on which the pressure is applied: $\phi=\mu r \omega P$. However, this heat flux is very unstationary on a brake disc due to thermoelastic instabilities (TEI) [11] and fading phenomenon [30] (i.e. drop of the friction coefficient for high temperatures) so that a more global definition of the heat flux derived from an energetic framework is preferred.

Mechanical stresses due to the caliper are deemed negligible with respect to the thermal stresses, namely, the pressure of the pads on the disc results from the hydraulic actuator, which rarely exceeds 80 bar, leading to compressive stresses of about a few MPa. As a consequence, the model used for braking simulations is an angular sector (Figure 2(b)) on which an axisymmetric flux is applied.

A test bench with inertia $J$ spinning with an initial velocity $\omega_{0}$ is considered on which braking with constant deceleration (i.e. the speed decreases linearly during braking) can be carried out several times. Thus, if $\Delta t$ is the braking duration, the angular velocity $\omega$ at time $t$ reads

$$
\omega(t)=\omega_{0}\left(1-\frac{t}{\Delta t}\right)
$$

The kinetic energy is then deduced at time $t$

$$
E_{k}(t)=\frac{1}{2} J \omega(t)^{2}=\frac{1}{2} J \omega_{0}^{2}\left(1-\frac{t}{\Delta t}\right)^{2}
$$

It is considered that all the kinetic energy is converted into heat and that 95 $\%$ of the latter is transmitted to the disc, while the remaining $5 \%$ is transmitted to the linings. The heat flux transmitted to the disc is then equal to the derivative of the kinetic energy with respect to time, weighted by a factor $\rho=0.95$. On the contact area with each pad, the expression of heat flux per unit area as a function of time $t$ then reads

$$
\phi(t)=-\rho \frac{1}{S_{p}} \frac{d E_{k}}{d t}=\rho \frac{J \omega_{0} \omega(t)}{S_{p} \Delta t}
$$

where $S_{p}$ is the contact surface between the pad and the disc. For an axisymmetric flux modeling, $S_{p}$ corresponds to the whole frictional surface

$$
S_{p}=\pi\left(R_{o u t}^{2}-R_{\text {in }}^{2}\right)
$$

where $R_{\text {in }}$ et $R_{\text {out }}$ are the inner and outer friction surface radii.

\subsection{Implementation strategy}

An implementation strategy of the three-dimensional model presented in Section 2 is proposed. As already mentioned, the main difficulties pertain to the management of the damage directions when solving the problem through a UMAT routine.

\subsubsection{Principal directions and damage basis}

The prerequisite is that the damage directions do not change with time. As damage occurs in planes normal to the eigen directions, it requires that the eigen directions must also remain unchanged. In order to check that hypothesis, a braking simulation was carried out with the proposed constitutive model without damage. 
The eigenvector associated with time $t$ corresponding to the maximum eigen stress is calculated. This vector $\boldsymbol{n}$ corresponds to the normal to the plane that will experience the most part of induced damage. The traction $\boldsymbol{T}=\boldsymbol{\sigma} \cdot \boldsymbol{n}$ is then calculated for all times. If the 1-component of $\boldsymbol{T}$ is always equal to the maximum eigen stress, it means that the eigen directions do not change with time.

Figure 5 shows for an element in the groove the eigen stresses $\sigma_{I}, \sigma_{I I}$ and $\sigma_{I I I}$ as functions of the 1-component of $\boldsymbol{T}$. The latter exactly corresponds to $\sigma_{I}$ for positive values, then is successively equal to $\sigma_{I I}$ and $\sigma_{I I I}$. This means that the eigen directions do not change, but the magnitude of the eigen stresses does change for negative values. The choice made in Section 2.2.1 for the representation of anisotropic damage is therefore validated.

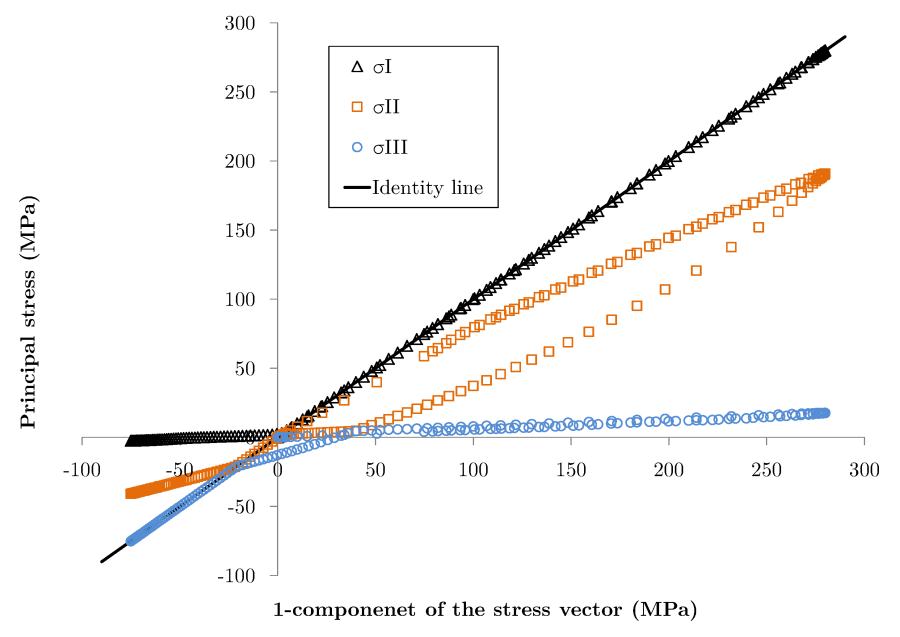

Fig. 5: Eigen stresses versus 1-component of the traction on the critical plane

\subsubsection{Damage direction management in ABAQUS}

From a numerical point of view, a major difficulty arises from the fact that the UMAT input data $\Delta \boldsymbol{\epsilon}$ are defined in the global basis. The latter is necessarily different from the eigen (or damage) basis of each element. As the numerical solution must take place in the stationary damage basis, a specific procedure was developed in the UMAT routine (Figure 6):

- During the first increment, the eigen basis $\mathbf{e}_{\mathbf{i}} \otimes \mathbf{e}_{\mathbf{j}}$ is defined once and for all. The transformation matrix $P_{i j}=\mathbf{e}_{\mathbf{i}} \cdot \mathbf{E}_{\mathbf{j}}$ is also defined, where $\mathbf{E}_{\mathbf{j}}$ corresponds to the global basis.

- The first increment is treated in the global basis, which is of no consequence as the damage is initialized to zero in all directions.

- During the second increment, the strain tensor increment $\Delta \epsilon^{D}$ and the stress tensor $\boldsymbol{\sigma}^{D}$ are defined in the damage basis $\mathbf{e}_{\mathbf{i}} \otimes \mathbf{e}_{\mathbf{j}}$ such that $\Delta \epsilon_{k l}^{D}=P_{k i}^{T} \Delta \epsilon_{i j} P_{j l}$ and $\sigma k l^{D}=P_{k i}^{T} \sigma_{i j} P_{j l}$. 
- The problem is solved in the damage basis.

- After convergence of Newton's scheme and updating of the stress and internal variables, an inverse basis change is carried out, from $\boldsymbol{\sigma}^{D}$ toward $\boldsymbol{\sigma}$, in order to enable ABAQus to reach global equilibrium. The internal variables are stored in the damage basis in the UMAT routine.

- From the third increment onward, the steps are those described for the second one.

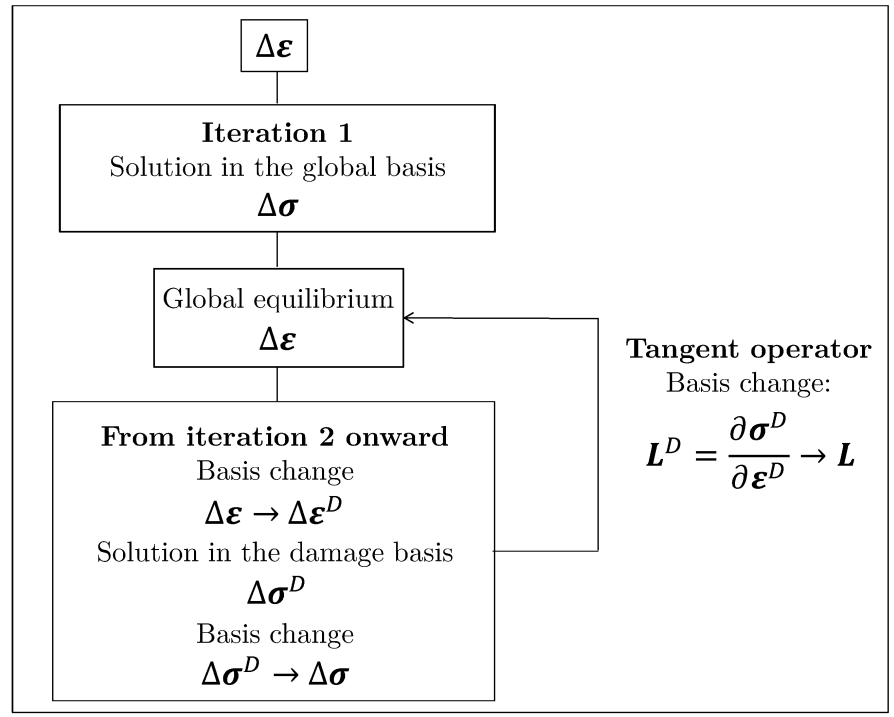

Fig. 6: Block diagram of the basis change procedure in the UMAT routine

As shown in Equation (60), the tangent operator $\boldsymbol{L}$ is calculated as $\boldsymbol{L}=\tilde{\mathbb{C}}: \boldsymbol{J}_{e e}^{*}$, such that it is defined in the damage basis, and denoted by $\boldsymbol{L}^{\boldsymbol{D}}$. In order to ensure global convergence, this matrix must be defined in the global basis of the finite element model. It is therefore necessary to operate a basis change, from the damage basis toward the structure basis. On a fourth order tensor, this operation is time consuming and Bond matrices [3] are preferred.

Matrix $\boldsymbol{P}$ defined above reads

$$
\boldsymbol{P}=\left(\begin{array}{lll}
a_{11} & a_{12} & a_{12} \\
a_{21} & a_{22} & a_{23} \\
a_{31} & a_{32} & a_{33}
\end{array}\right)
$$


Similarly, $\boldsymbol{M}$ and $\boldsymbol{N}$ matrices are defined as

$$
\boldsymbol{M}=\left(\begin{array}{cccccc}
a_{11}^{2} & a_{12}^{2} & a_{13}^{2} & 2 a_{12} a_{13} & 2 a_{13} a_{11} & 2 a_{11} a_{12} \\
a_{21}^{2} & a_{22}^{2} & a_{23}^{2} & 2 a_{22} a_{23} & 2 a_{23} a_{21} & 2 a_{21} a_{22} \\
a_{31}^{2} & a_{32}^{2} & a_{33}^{2} & 2 a_{32} a_{33} & 2 a_{33} a_{31} & 2 a_{31} a_{32} \\
a_{21} a_{31} & a_{22} a_{32} & a_{23} a_{33} & a_{22} a_{33}+a_{23} a_{32} & a_{21} a_{33}+a_{23} a_{31} & a_{22} a_{31}+a_{21} a_{32} \\
a_{31} a_{11} & a_{32} a_{12} & a_{33} a_{13} & a_{12} a_{33}+a_{13} a_{32} & a_{13} a_{31}+a_{11} a_{33} & a_{11} a_{32}+a_{12} a_{31} \\
a_{11} a_{21} & a_{12} a_{22} & a_{13} a_{23} & a_{12} a_{23}+a_{13} a_{22} & a_{13} a_{21}+a_{11} a_{23} & a_{11} a_{22}+a_{12} a_{21}
\end{array}\right)
$$

and

$\boldsymbol{N}=\left(\begin{array}{cccccc}a_{11}^{2} & a_{12}^{2} & a_{13}^{2} & a_{12} a_{13} & a_{13} a_{11} & a_{11} a_{12} \\ a_{21}^{2} & a_{22}^{2} & a_{23}^{2} & a_{22} a_{23} & a_{23} a_{21} & a_{21} a_{22} \\ a_{31}^{2} & a_{32}^{2} & a_{33}^{2} & a_{32} a_{33} & a_{33} a_{31} & a_{31} a_{32} \\ 2 a_{21} a_{31} & 2 a_{22} a_{32} & 2 a_{23} a_{33} & a_{22} a_{33}+a_{23} a_{32} & a_{21} a_{33}+a_{23} a_{31} & a_{22} a_{31}+a_{21} a_{32} \\ 2 a_{31} a_{11} & 2 a_{32} a_{12} & 2 a_{33} a_{13} & a_{12} a_{33}+a_{13} a_{32} & a_{13} a_{31}+a_{11} a_{33} & a_{11} a_{32}+a_{12} a_{31} \\ 2 a_{11} a_{21} & 2 a_{12} a_{22} & 2 a_{13} a_{23} & a_{12} a_{23}+a_{13} a_{22} & a_{13} a_{21}+a_{11} a_{23} & a_{11} a_{22}+a_{12} a_{21}\end{array}\right)$

Definitions (66) and (67) imply that $\boldsymbol{N}^{-1}=\boldsymbol{M}^{T}$, which helps defining the tangent matrix $\boldsymbol{L}$ in the global basis

$$
\boldsymbol{L}=\boldsymbol{M} \boldsymbol{L}^{\boldsymbol{D}} \boldsymbol{N}^{T}
$$

\subsection{Stress/strain paths in the structure}

With the constitutive model previously described it is possible to carry out braking simulations in which stress/strain paths in critical areas (i.e. the groove and the friction bands) are studied. Two configurations were tested, namely, the first one is a small plain brake disc 266-mm in diameter (denoted D266) mounted on a light vehicle; the second one is a large ventilated disc 330-mm in diameter (denoted D330) mounted on a much heavier vehicle.

A series of severe brakings (i.e. deceleration from $80 \% V_{\max }$ to $40 \% V_{\max }$ at $0.5 \mathrm{~g}$ ) was simulated for the two configurations. The constitutive model accounts for anisotropic damage but without flake closure. Stress/strain paths in the radial and hoop directions in the groove are shown in Figure 7(a). The response is quickly characterized by plastic shakedown. The "coning" of the disc during the first braking induces a global deformation of the friction ring and permanent strains in the groove. As a result, even at the end of the cooling phase, there is still a positive residual strain. Under these conditions, flake closure will never occur in the groove and the present constitutive model is sufficient to describe the actual behavior of that area.

The strong thermal gradient on the friction bands gives rise to compressive stresses and plasticity during braking, and residual tensile stresses after cooling (Figure 7(b)). The latter ones are below the threshold (about $90 \mathrm{MPa}$ ) at which damage occurs [2]. This result means that in the friction bands graphite flakes do not debond, and damage is equal to zero in all directions. Again, the present constitutive model is sufficient to describe the actual behavior of the studied area.

Outside these critical areas, no other part of the disc requires flake closure be taken into account; the flakes are either debonded or closed, and remain in that state. For this disc configuration and the considered braking conditions, the 


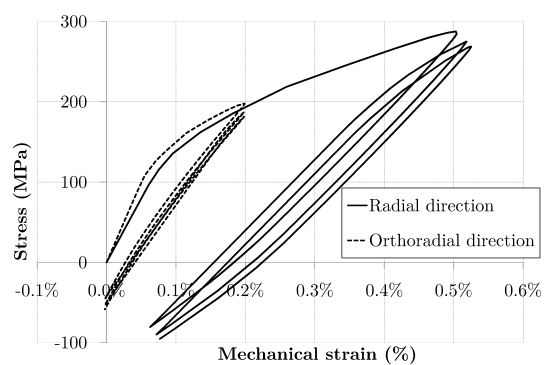

(a) Groove

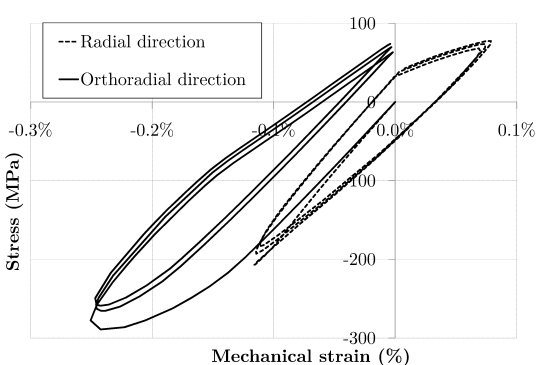

(b) Friction band

Fig. 7: Stress/strain paths on the D266 disc without damage

present constitutive model is therefore sufficient to describe the actual behavior of the entire structure.

Conclusions are rather different for D330 brake disc, for which the applied thermal flux is more important, owing to the higher inertia of the corresponding vehicle. The stress/strain path on the friction bands shown in Figure 8 with the current constitutive model shows a non-physical drift of the mechanical response toward positive stresses. This phenomenon is explained by the following reasons:

- the tensile residual stress after the first cooling is greater than $90 \mathrm{MPa}$, damage is therefore activated in this direction;

- as a consequence, Hooke's tensor is affected by damage;

- given that no closure condition or damage deactivation is considered, components of Hooke's tensor remain underestimated, resulting in an overestimation of the minimum compressive stress reached at the end of the braking phase;

- the subsequent tensile stress is thus overestimated, damage increases and components of Hooke's tensor decrease;

- the process intensifies for each braking and results in a totally non physical response.

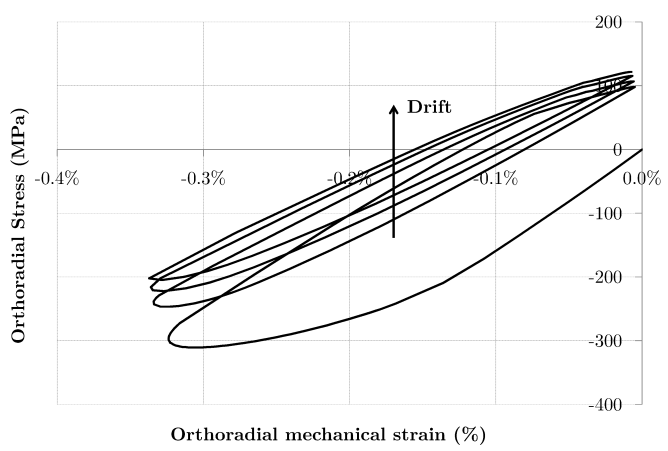

Fig. 8: Stress/strain path on the $D 330$ friction band without closure condition 
The solution chosen to counteract this phenomenon consists of a damage deactivation strategy. The procedure described in Section 3.6 is applied on elements for which a mechanical strain value of $-0.2 \%$ is observed. This choice is made because the friction bands alone are concerned, namely, the only area where this value is reached. Moreover it is assumed that only severe brakings will cause damage in tension, resulting in large negative mechanical strains.

\subsection{Damage deactivation}

The damage deactivation process consists of deactivating damage instantaneously when flakes close. Consequently, it must be treated direction by direction. As shown in Ref. [2], flake closure occurs approximately at the strain level at which the flakes opened. The strain level is easy to define with the 1D two-branch model, but there is no direct way to define it with the above $3 \mathrm{D}$ model. However, for the loading considered in the friction bands, initial flake debonding (i.e. for the first braking) and subsequent flake openings (i.e. for subsequent brakings) are most likely to occur when the stress shifts from negative to positive levels, at a corresponding strain level $\epsilon_{t}$. This condition defines activation and the deactivation condition can therefore be defined as the shift from $\epsilon>\epsilon_{t}$ to $\epsilon<\epsilon_{t}$.

Such activation and deactivation conditions were tested with unsatisfactory results (Figure 9). Simple damage deactivation does not physically represent the mechanisms involved, namely, the material actually includes two separate phases represented by the two-branch rheological model [2]. Deactivating damage instantaneously upon unloading thus results in non-smooth paths and non physical behaviors with a drift even more pronounced than in the undamaged case. This bilinear path is a typical inconsistency associated with damage activation/deactivation conditions. More realistic models based on continuous damage deactivation are proposed, in which microcracks close gradually [19]. Yet the specific conditions under which flakes open and close on a 3D structure make the application of such approaches quite complex in the present case.

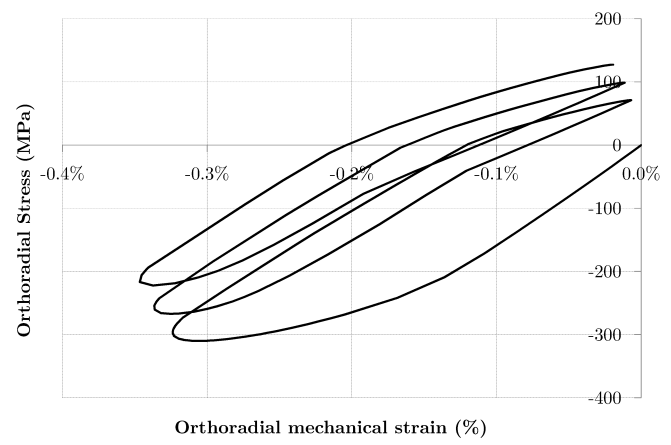

Fig. 9: Stress/strain path in the $D 330$ friction band with damage deactivation at $\sigma=0$ 
The solution finally adopted to counteract this phenomenon is to enforce damage deactivation always at the same strain level, thereby avoiding drift. The simplest and most pragmatic choice is to deactivate damage as early as the first unloading increment. This condition makes no sense on fully reversed cyclic tests and will result in a very bad description of the overall unloading behavior. Conversely, the error is minimal on the friction band (i.e. the only area where this condition is in fact activated), because subsequent closure occurs very quickly after unloading. The corresponding stress path is shown in Figure 10 where a stabilized behavior is observed, a reasonable maximum stress estimation and a low error during unloading.

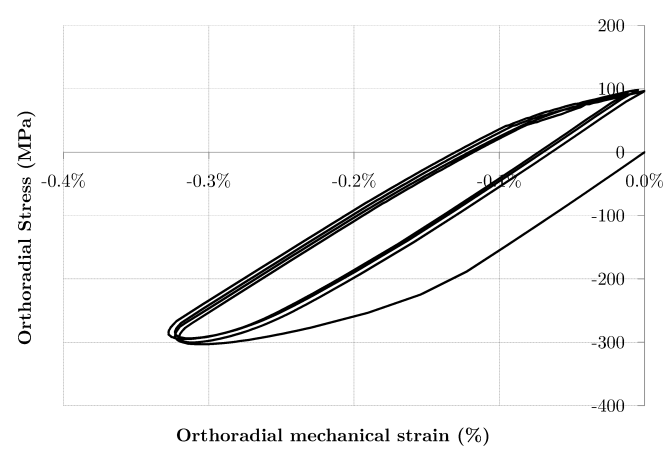

Fig. 10: Stress/strain path in the $D 330$ friction band with damage deactivation upon early unloading

\section{Conclusions}

The present paper describes an attempt to generalize a one-dimensional setting [2] to describe the thermomechanical behavior of flake graphite cast iron to a three dimensional constitutive law valid for complex loadings on a brake disc. Directly transposing the $1 \mathrm{D}$ model to a 3D model was examined but proved to be inconsistent. Consequently, a pragmatic solution was proposed (Section 2.2), which is based on the following points:

- No area other than the friction bands experiences flake opening/closure.

- Damage deactivation is introduced on the friction bands.

- The opening condition is defined by $\sigma \geq 0$ in the considered direction.

- Flake closure is enforced at the very beginning of the unloading phase.

The model allows an accurate simulation of the mechanical behavior in the groove. In the friction bands, the asymptotic global behavior is also well described. However, a small error during the unloading phase, which is inherent to the damage deactivation procedure, still exists. This error gives rise to a small overestimation of the mechanical energy dissipated during a braking cycle.

This model must now be tested on a large number of brake discs with varied designs to be completely validated. Low cycle fatigue criteria, based on quantities 
such as mechanical dissipated energy will also be evaluated, in order to work out a complete brake disc fatigue design framework.

Acknowledgements This work was carried out within the Executive Doctorate in Industry framework, and was supported by PSA Peugeot Citroën.

\section{References}

1. Abdi, R.E., Samrout, H.: Effet de la distribution du flux dans un disque de frein sous sollicitations thermomécaniques. Mécanique Industrielle et Matériaux 52 (1999)

2. Augustins, L., Billardon, R., Hild, F.: Constitutive model for flake graphite cast iron automotive brake discs. From macroscopic multiscale models to a 1D rheological description. Contin. Mech. Thermodyn. (2015)

3. Auld, B.A.: Acoustic Fields and Waves in Solids, pp. 74-75. Robert E. Krieger Publishing Company (1990)

4. Bagnoli, F., Dolce, F., Bernabei, M.: Thermal fatigue cracks of fire fighting vehicles gray iron brake discs. Eng. Fail. Anal. 16, 152-163 (2009)

5. Besson, J.: Implementation of material constitutive equations in finite element codes. http://mms2.ensmp.fr/msi_paris/nlfe/transparents/paris_tech2005b.pdf. Accessed: 2013-09-30

6. Chaboche, J.L.: Sur l'utilisation des variables d'état internes pour la description du comportement viscoplastique et de la rupture par endommagement. Symp. Franco-Polonais de Rhéologie et mécanique (1977)

7. Chaboche, J.L.: Description phénoménologique de la viscoplasticité cyclique avec endommagement. Thèse d'Etat, Université Pierre et Marie Curie, Paris 6 (1978)

8. Chaboche, J.L.: Le concept de contrainte effective appliquée à l'élasticité et à la viscoplasticité en présence d'un endommagement anisotrope. Mech. Behav. of Anisotropic Solids Proc., Euromech 115 pp. 737-760 (1979)

9. Chow, C.L., Wang, J.: An anisotropic theory of continuum damage mechanics for ductile fracture. Int. J. Fract. 27, 547-558 (1987)

10. Cordebois, J.P., Sidoroff, F.: Endommagement anisotrope en élasticité et plasticité. Journal de Mécanique Théorique et Appliquée Numéro spécial, 45-60 (1982)

11. Cristol, A.L., Desplanques, Y., Osterle, W., Degallaix, G.: Coupling between thermal localisation and friction mechanisms: heat accumulation effect. Proceedings of the 6th European Conference on Braking pp. 61-69 (2010)

12. D'Cruz, A.H.: Surface crack initiation in ventilated disc brakes under transient thermal loading. Institution of Mech. Eng. C382/05 (1989)

13. Denoual, C., Hild, F.: A damage model for the dynamic fragmentation of brittle solids. Comp. Meth. Appl. Mech. Eng. 183, 247-258 (2000)

14. Desmorat, R., Gatuingt, F., Ragueneau, F.: Non local anisotropic damage model and related computational aspects for quasi-brittle materials. Eng. Fract. Mech. 74, 15391560 (2007)

15. Dufrenoy, P.: Etude du comportement thermomécanique des disques de freins vis à vis des risques de défaillance. application au domaine ferroviaire. $\mathrm{Ph}$. D. thesis, Université des sciences et technologies de Lille (1995)

16. Dufrenoy, P., Bodovillé, G., Degallaix, G.: Damage mechanisms and thermomechanical loading of brake discs. European Struc. Integr. Soc. 29, 167-176 (2002)

17. Fabrikant, V.I.: Complete solutions to some mixed boundary value problems in elasticity. Advances in Appl. Mech 27, 153-223 (1990)

18. Forquin, P., Hild, F.: A probabilistic damage model of the dynamic fragmentation process in brittle materials. Adv. Appl. Mech. 44, 1-72 (2010)

19. Ganczarski, A., Cegielski, M.: Damage deactivation of engineering materials ans structures. In: H. Altenbach, S. Kruch (eds.) Advanced Materials Modelling for Structures, pp. 153163. Springer Verlag, Berlin Heildelberg (2013)

20. Germain, P.: Cours de mécanique des milieux continus. Masson, Paris (1973)

21. Gilbert, G.: An evaluation of the stress-strain properties of flake graphite cast iron in tension and compression. J Br. Cast Iron Res. Assoc. 7, 745-789 (1959) 
22. Halm, D., Dragon, A.: A model of anisotropic damage by mesocrack growth; unilateral effect. Int. J. Damage Mech. 5, 384-402 (1996)

23. Kachanov, L.M.: Continuum model of medium with cracks. J. Eng. Mech. Divis. 106, 1039-1051 (1980)

24. Krajcinovic, D.: Damage Mechanics. North Holland (1996)

25. Leckie, F.A., Onat, E.T.: Tensorial nature of damage measuring internal variables. Int. Union of Theor. and Appl. Mech. Symp., Phys. Nonlinearities in Struct. Anal. pp. 140-155 (1981)

26. Lee, S., Yeo, T.: Temperature and coning analysis of brake rotor using axisymmetric finite element technique. Sci. Technol. 3, 17-22 (2000)

27. Lemaitre, J., Chaboche, J.L.: Aspect phénoménologique de la rupture par endommagement. Journal de Mécanique Appliquée 3, 317-365 (1978)

28. Lemaitre, J., Chaboche, J.L.: Mécanique des Matériaux Solides, second edition. Dunod, Paris (1996)

29. Lemaitre, J., Desmorat, R.: Eng. Damage Mech. Springer, New York (2005)

30. Limpert, R.: Brake design and safety, second ed. SAE Inernational (1999)

31. Lubarda, V.A., Krajcinovic, D.: Damage tensors and crack density distribution. Int. J. Solids Struct. 30, 2859-2877 (1993)

32. Marquis, D.: Phénoménologie et thermodynamique - Application au couplage entre la thermoélasticité, la plasticité, le vieillissement et l'endommagement. Thèse d'Etat, Université Pierre et Marie Curie, Paris 6 (1989)

33. Murakami, S., Ohno, A.: A continuum theory of creep and creep damage. Int. Union of Theor. and Appl. Mech. pp. 422-444 (1981)

34. Ortiz, M.: A constitutive theory for the inelastic behavior of concrete. Mech. Mater. 4, 67-93 (1985)

35. Ortiz, M., Popov, E.P.: Accuracy and stability of integration algorithms for elastoplastic constitutive relations. Int. J. Num. Methods Eng. 21, 1561-1576 (1985)

36. Petry, C.: Caractérisation et modélisation du comportement et de l'endommagement d'alliages métalliques sur une grande plage de température. Ph.D. thesis, Ecole Normale Supérieure de Cachan (2006)

37. Simulia: Abaqus/Strandard subroutines. Abaqus 6.13 User Subroutines Reference Manual Section 1.1 (2013)

38. Skrzypek, J., Ganczarski, A.: Application of the orthotropic damage growth rule to variable principal directions. Int. J. Damage Mech. 7, 180-206 (1998)

39. Vallet, F.: Etude de la fissuration d'un disque de frein à partir de l'analyse de son comportement thermomécanique. Ph.D. thesis, INSA Lyon (1999) 